\title{
The Euler characteristic of the configuration space of planar spidery linkages
}

\author{
YASUHIKO KAMIYAMA \\ SHUICHI TSUKUDA
}

\begin{abstract}
Among planar mechanical linkages, polygonal and spidery linkages are particularly important. We define a planar linkage which contains them. Constructing a Bott-Morse function on the configuration space of this, we determine the Euler characteristic. In particular, we give a new calculation of the Euler characteristic of polygon spaces.
\end{abstract}

58E05; 57M20

\section{Introduction}

The topology of configuration spaces of planar mechanical linkages has been studied by many people. Among these linkages, polygonal and spidery ones are particularly important. We call the configuration space of a polygonal linkage a polygon space. The study of polygon spaces was originated by Hausmann [7], Kapovich and Millson [10] and Walker [15]. Their study has a long history and has begotten many references. For example, the homology groups of polygon spaces of arbitrary edge lengths have been determined by Farber and Schütz [6].

A linkage has $n$ arms such that each arm has two legs and each leg has length $\ell$. The linkage also has a rotational joint in the middle, and the endpoints of the arms are fixed to the vertices of $P_{n}$, where $P_{n}$ denotes the regular $n$-gon in $\mathbb{C}$. We assume that its arms and joints can intersect each other. Thus, a spidery linkage is a planar 2-leg machine. Let $M_{n}(\ell)$ be its configuration space.

The main result of Shvalb, Shoham and Blanc's [14] states that when $\ell$ is small, $M_{n}(\ell)$ is diffeomorphic to a connected closed orientable surface of genus $(n-4) 2^{n-3}+1$. (See Example 2.6(ii) for more details.) To prove this, giving a cellular decomposition, one computes $\chi\left(M_{n}(\ell)\right)$. The argument essentially shows a diffeomorphism between $M_{n}(\ell)$ and $\mathcal{Z}_{P_{n}}\left(S^{0}\right)$, the moment-angle complex of $S^{0}$ with respect to $P_{n}$.

We recall the history of the study of the moment-angle complex. For a space $X$ and a convex polytope $P$, let $\mathcal{Z}_{P}(X)$ be the moment-angle complex of $X$ with respect to $P$. 
The complex was first introduced for $X=S^{0}$ and $S^{1}$ in order to study toric manifolds. (See Davis and Januszkiewicz [5].) In particular, it was shown that $\mathcal{Z}_{P_{n}}\left(S^{0}\right)$ is a connected closed orientable surface of genus $(n-4) 2^{n-3}+1$. It is to be noted that before [14], we did not expect that moment-angle complexes appeared as configuration spaces of linkages.

Many homological properties of $\mathcal{Z}_{P}(X)$ have been studied; see, for example, Buchstaber and Panov [4]. Bahri, Bendersky, Cohen and Gitler [1] observed that $\mathcal{Z}_{P}(X)$ has an important property from the viewpoint of the homotopy theory, that is, there is a natural splitting of $\Sigma \mathcal{Z}_{P}(X)$.

Related papers to [14] are given as follows: First, O'Hara [13] proved that if $\ell$ is median, then $M_{n}(\ell)$ is a singular space and if $\ell$ is large, then $M_{n}(\ell)$ is diffeomorphic to a connected closed orientable surface of genus $(5 n-4) 2^{n-3}+1$. He proved this by constructing a Morse function on $M_{n}(\ell)$. In particular, when $\ell$ is small, the Morse function induces the cellular decomposition in [14].

Second, in [9], we generalized the planar 2-leg machine to one in $\mathbb{R}^{d}$. There it was shown that the configuration space is homeomorphic to $\mathcal{Z}_{P_{n}}\left(S^{d-1}\right)$ and that $\Sigma \mathcal{Z}_{P_{n}}\left(S^{d-1}\right)$ is a bouquet of spheres. Namely, a special case of the splitting in [1] was already given in [9].

In this paper, we generalize the planar 2-leg machine to an $m$-leg machine. We denote by $M(n, m, \ell)$ its configuration space. The space $M(n, m, \ell)$ contains the above spaces; the case $n=2$ is the polygon space and $m=2$ is $M_{n}(\ell)$. But the space $M(n, m, \ell)$ is much more complicated than $M(n, 2, \ell)$ because the topological type of polygon spaces with all the edge lengths but one equal varies greatly. The purpose of this paper is to determine $\chi(M(n, m, \ell))$ using Bott-Morse theory.

The key to proving our main result is as follows: Recall that Hausmann [7] and Walker [15] computed the indices of a Morse function on the space of free robot arms (see also Theorem 3.3). The result is important and used effectively in [6]. We generalize their result to polygon spaces (see Theorem 4.2).

This paper is organized as follows. In Section 2 we state our main theorems. In Section 3 we summarize the previous results. In Section 4 we generalize the results in $[7 ; 15]$. In Section 5 we prove the main theorems. In Section 6, we consider the case $n=2$ and give a new proof of the Euler characteristic formula for polygon spaces.

Because of the nature of the subject, we use many symbols, subscripts and superscripts. For the reader's convenience, we present some notational conventions here. In principle, we use the letters 
- $\quad \mu, v, i, j, k, l, m, n, N$ for nonnegative or positive integers,

- $\ell, c, d, r, s$ for real numbers,

and, for some positive integer $N \geq 1$, we write

- $\quad a, b, z, w$ for elements of $T^{N}$,

- $u$ for elements of $\mathbb{C}^{N}$ and

- $\varepsilon, \delta$ for elements of $\{-1,1\}^{N}$.

A superscript $k$ stands for the $k^{\text {th }}$ coordinate of an element of $\{-1,1\}^{N}, T^{N}$ or $\mathbb{C}^{N}$, that is, say, $a_{i}^{k}$ stands for the $k^{\text {th }}$ coordinate of an element $a_{i}=\left(a_{i}^{1}, \ldots, a_{i}^{N}\right) \in T^{N}$. Before we leave this section, we give a remark: in order to simplify the notation, we consider the situation that all legs have lengths $\ell$ and each arm has $m$ legs in this paper. But the results and proofs in this paper remain valid under the generalizations that the anchors $v_{i}$ are arranged at any points in $\mathbb{C}$, the arms have varying number of legs and the legs have varying length.

Acknowledgements The authors are grateful to the referee for valuable comments, in particular for catching an error in the proof of Theorem D in the original version.

\section{The main theorems}

Definition 2.1 $\quad$ (i) We fix an integer $n \geq 2$ and define

$$
v_{i}=\cos \frac{2(i-1) \pi}{n}+\sqrt{-1} \sin \frac{2(i-1) \pi}{n} .
$$

(ii) We fix an integer $m \geq 2$ and $\ell \in \mathbb{R}_{>0}$. We write an element of $\mathbb{C}^{m-1}$ as $u=\left(u^{1}, \ldots, u^{m-1}\right)$. We define

$$
\begin{array}{r}
M(n, m, \ell)=\left\{\left(u_{1}, \ldots, u_{n}, q\right) \in\left(\mathbb{C}^{m-1}\right)^{n} \times \mathbb{C}|| u_{i}^{1}-v_{i}|=| u_{i}^{k+1}-u_{i}^{k}|=| q-u_{i}^{m-1} \mid=\ell\right. \\
\text { for all } 1 \leq i \leq n \text { and } 1 \leq k \leq m-2\} .
\end{array}
$$

Thus $M(n, m, \ell)$ is the configuration space of a spidery linkage obtained by joining $n$ arms such that each arm consists of $m$ legs of length $\ell$. (See Figure 1.)

For generic $\ell, M(n, m, \ell)$ is a connected closed orientable manifold of dimension $n(m-2)+2$. (See Definition 2.8 and Theorem A.) It is clear that $M(n, m, \ell)=\varnothing$ if $\ell<1 / m$ and $M(n, m, \ell)=$ one point $\}$ if $\ell=1 / m$. 


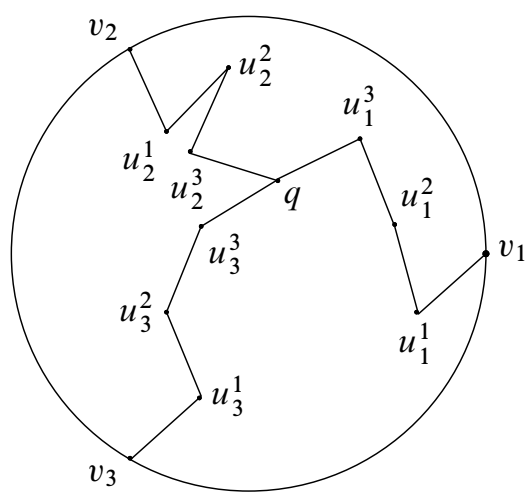

Figure 1: A linkage in $M(3,4, \ell)$

By setting $a_{i}^{k}=\left(u_{i}^{k}-u_{i}^{k-1}\right) / \ell$ (where we set $u_{i}^{0}=v_{i}$ and $u_{i}^{m}=q$ ), we can identify $M(n, m, \ell)$ with the space

$$
\left\{\left(a_{1}, \ldots, a_{n}\right) \in\left(T^{m}\right)^{n} \mid v_{i}+\ell \sum_{k=1}^{m} a_{i}^{k}=v_{j}+\ell \sum_{k=1}^{m} a_{j}^{k} \text { for all } 1 \leq i, j \leq n\right\} .
$$

Hereafter, we identify $M(n, m, \ell)$ with this space.

Definition $2.2 \quad$ (i) Let $1 \leq i \leq n$. We define a map $p_{i}: M(n, m, \ell) \rightarrow T^{m}$ by

$$
p_{i}\left(a_{1}, \ldots, a_{n}\right)=a_{i}
$$

(ii) We define a map $q: M(n, m, \ell) \rightarrow \mathbb{C}$ by

$$
q\left(a_{1}, \ldots, a_{n}\right)=v_{1}+\ell \sum_{k=1}^{m} a_{1}^{k}
$$

Definition 2.3 (i) For an integer $n \geq 2$, let $d_{n}$ be the maximum distance between $n^{\text {th }}$ roots of unity. More precisely, we set

$$
d_{n}= \begin{cases}2 & n \text { is even } \\ 2 \cos \frac{\pi}{2 n} & n \text { is odd }\end{cases}
$$

(ii) For integers $m, n \geq 2$, we define

$$
b(n, m)=\frac{(-m+2)\left(1+\cos \frac{\pi}{n}\right)+2 \sqrt{m^{2} \cos ^{4} \frac{\pi}{2 n}+(m-1) \sin ^{2} \frac{\pi}{n}}}{4(m-1)}
$$

and we set

$$
\xi(n, m)= \begin{cases}1 /(m-1) & n \text { is even } \\ b(n, m) & n \text { is odd }\end{cases}
$$


Remark 2.4 We will see later that $d\left(v_{i}, q(M(n, m, \ell))\right)=\ell(m-2)$ precisely when $\ell=\xi(n, m)$. (See Section 5.)

Definition 2.5 For $(N, r) \in \mathbb{N} \times \mathbb{R}_{>0}$ with $N \geq 3$, we define

$$
\begin{array}{r}
P(N, r)=\left\{\left(u_{1}, \ldots, u_{N}\right) \in \mathbb{C}^{N} \mid u_{1}=-r / 2, u_{N}=r / 2\right. \text { and } \\
\left.\left|u_{i+1}-u_{i}\right|=1 \text { for all } 1 \leq i \leq N-1\right\} .
\end{array}
$$

Consider the space of $N$-gons in $\mathbb{C}$ with edge lengths $(1, \ldots, 1, r)$. The orientationpreserving isometry group naturally acts on it and the orbit space is identified with $P(N, r)$. Hence $P(N, r)$ is the configuration space of polygons as considered in Hausmann [7] and Kapovich and Millson [10].

Example 2.6 (i) There is a homeomorphism

$$
M(2, m, \ell) \cong P(2 m+1,2 / \ell) .
$$

Thus our linkage produces $P(2 m+1, r)$ for all $r>0$.

(ii) In Section 1 , we denoted by $M_{n}(\ell)$ the configuration space of spidery linkage which was defined in Shvalb, Shoham and Blanc [14]. In our notation, $M_{n}(\ell)$ is $M(n, 2, \ell)$. Then the main results in O'Hara [13] and [14] are that if $\ell \neq d_{n} / 2$, then $M(n, 2, \ell)$ is a connected closed orientable surface such that

$$
\chi(M(n, 2, \ell))= \begin{cases}(4-n) 2^{n-2} & \frac{1}{2}<\ell<d_{n} / 2, \\ (4-5 n) 2^{n-2} & d_{n} / 2<\ell .\end{cases}
$$

Definition 2.7 (i) Let $e_{i}$ be the segment through $v_{i}$ and $v_{i+1}$, where $v_{i}$ is defined in Definition 2.1(i). When $n \geq 3$, let $P_{n}$ be the regular $n$-gon $P_{n}$ in $\mathbb{C}$ whose edges are $e_{1}, \ldots, e_{n-1}$ and $e_{n}$. In the case $n=2$, we think of $P_{2}$ as the 2 -gon, namely, a shape with 2 vertices and 2 edges and positive area, as can be realized on the surface of a sphere.

(ii) For a based space $X$, we define a moment-angle complex by

$$
\mathcal{Z}_{P_{n}}(X)=\left\{\left(x_{1}, \ldots, x_{n}, q\right) \in X^{n} \times P_{n}\right\} / \sim,
$$

where the equivalence relation $\sim$ is generated by the relation

$$
\left(x_{1}, \ldots, x_{n}, q\right) \sim\left(x_{1}, \ldots, *, \ldots, x_{n}, q\right) \quad \text { if } q \in e_{i} .
$$

Definition 2.8 Let $m, n \geq 2$ be integers.

(i) We say that the $i^{\text {th }}$ arm of a configuration $\left(a_{1}, \ldots, a_{n}\right) \in M(n, m, \ell)$ is aligned if $a_{i}^{k}= \pm a_{i}^{1}$ for all $1 \leq k \leq m$. 
(ii) Let $\ell \in \mathbb{R}$ be a real number such that $\ell \geq 1 / m$. We say $\ell$ is good with respect to $m$ and $n$ if each configuration in $M(n, m, \ell)$ has at most two aligned arms and the aligned two arms are not colinear; otherwise, $\ell$ is said to be bad. In other words, $\ell$ is good if and only if:

(a) For each $\left(a_{1}, \ldots, a_{n}\right) \in M(n, m, \ell),\left|q\left(a_{1}, \ldots, a_{n}\right)-v_{i}\right| / \ell-m$ is an even integer for at most two $i$.

(b) If $v_{i}+k \ell\left(v_{i}-v_{j}\right) /\left|v_{i}-v_{j}\right| \in q(M(n, m, \ell))$ for some $k \equiv m(\bmod 2)$, then $\left|v_{i}-v_{j}\right| / \ell$ is not an even integer.

Note that there are only finitely many bad $\ell$ for fixed $m, n$.

Now we state our first main result.

Theorem A Fix $m, n \geq 2$. Let $\ell_{0}<\ell_{1}<\cdots<\ell_{\text {max }}$ be all the bad $\ell$ with respect to $m$ and $n$. Then we have the following:

(i) $\ell_{0}=1 / m, \ell_{1}=\xi(m, n)$ and

$$
\ell_{\max }= \begin{cases}d_{n} / 2 & m \text { is even } \\ 1 & m \text { is odd }\end{cases}
$$

(ii) If $\ell>1 / m$ is good, then $M(n, m, \ell)$ is a closed orientable manifold of dimension $n(m-2)+2$.

(iii) The diffeomorphism type of $M(n, m, \ell)$ is constant for $\ell \in\left(\ell_{i}, \ell_{i+1}\right)$ for all $0 \leq i \leq \max$, where $\ell_{\max +1}$ is understood to be $\infty$.

(iv) If $\ell \in\left(\ell_{0}, \ell_{1}\right)$, that is, if

$$
\frac{1}{m}<\ell<\xi(n, m)
$$

then $M(n, m, \ell)$ is homeomorphic to $\mathcal{Z}_{P_{n}}\left(S^{m-2}\right)$.

Remark 2.9 (i) Example 2.6(ii) is a special case of Theorem A for $m=2$.

(ii) Note that $d_{n} / 2=1$ if $n$ is even and $d_{n} / 2<1$ if $n$ is odd.

(iii) Note that $\xi(n, m)>1 / m$. Hence, for any $m, n \geq 2$, the moment angle complex $\mathcal{Z}_{P_{n}}\left(S^{m-2}\right)$ can be realized as a planar spidery linkage.

Hereafter, we mostly assume that $\ell$ satisfies the following conditions. 
Assumption 2.10 For given $m$ and $n, \ell$ is good with respect to $m$ and $n$. Moreover, $\ell$ satisfies the following conditions:

(i) If $q\left(a_{1}, \ldots, a_{n}\right)=0$, then no arm of $\left(a_{1}, \ldots, a_{n}\right)$ is aligned. In other words, $1 / \ell-m$ is not an even integer.

(ii) If the $i^{\text {th }}$ arm of $\left(a_{1}, \ldots, a_{n}\right)$ is aligned in the direction of $v_{i}$, then no other arms are aligned. In other words, if there exist $\left(a_{1}, \ldots, a_{n}\right) \in M(n, m, \ell)$ and $i$ such that $a_{i}^{k}= \pm v_{i}$ for all $1 \leq k \leq m$, then $\left|q\left(a_{1}, \ldots, a_{n}\right)-v_{v}\right| / \ell-m$ is not an even integer for all $v \neq i$.

Note that for a fixed $m$ and $n$, Assumption 2.10 excludes finitely many choices of $\ell$. Also note that if $\ell_{0}<\ell<\ell_{1}$ or $\ell>\ell_{\max }$, then $\ell$ satisfies this assumption.

Definition 2.11 Let $N$ be an arbitrary positive integer.

(i) For an element $u=\left(u^{1}, \ldots, u^{N}\right) \in \mathbb{C}^{N}$, we denote the complex number $\sum_{k=1}^{N} u^{k}$ by $\sigma(u)$.

(ii) For an element $u \in \mathbb{C}^{N}$ with $\sigma(u) \neq 0$, we define an element $e(u) \in T^{1}$ by

$$
e(u)=\sigma(u) /|\sigma(u)| \text {. }
$$

(iii) An element $u \in \mathbb{C}^{N}$ is said to be aligned if $u^{k} \neq 0$ and $u^{k} /\left|u^{k}\right|= \pm u^{1} /\left|u^{1}\right|$ for all $1 \leq k \leq N$.

(iv) For an aligned element $u=\left(u^{1}, \ldots, u^{N}\right) \in \mathbb{C}^{N}$ satisfying $\sigma(u) \neq 0$, we define a nonnegative integer $\mu(u)$ by

$$
\mu(u)=\sharp\left\{k\left|u^{k} /\right| u^{k} \mid=e(u)\right\}=\sharp\left\{k \mid u^{k} \overline{\sigma(u)}>0\right\} .
$$

Definition 2.12 Let $1 \leq i \leq n$ and $v=\left(z^{1}, \ldots, z^{m}\right) \in\left\{-v_{i}, v_{i}\right\}^{m}$.

We denote $v_{i}+\ell \sigma(v)=v_{i}+\ell \sum_{k=1}^{m} z^{k} \in \mathbb{C}$ by $q_{i, v}$.

We define a (possibly empty) subspace of $M(n, m, \ell)$ by

$$
R_{i, v}=p_{i}^{-1}(v)=\left\{\left(a_{1}, \ldots, a_{n}\right) \in M(n, m, \ell) \mid a_{i}=v\right\} .
$$

If $R_{i, v}$ is not empty, we set $r_{i, v, v}=\left|q_{i, v}-v_{v}\right| / \ell$. Then it is clear that

$$
R_{i, v} \cong \prod_{1 \leq \nu \leq n, v \neq i} P\left(m+1, r_{i, v, v}\right)
$$


Definition 2.13 Let $1 \leq i<j \leq n$ and $z, w \in T^{m}$ be aligned elements, namely, $z^{k}= \pm z^{1}$ and $w^{k}= \pm w^{1}$ for all $1<k \leq m$, satisfying

$$
v_{i}+\ell \sigma(z)=v_{j}+\ell \sigma(w) .
$$

We denote $v_{i}+\ell \sigma(z)=v_{j}+\ell \sigma(w) \in \mathbb{C}$ by $q_{i, j, z, w}$. Note that if $\ell$ is good and $z$ and $w$ satisfy the condition (2-3), then $\sigma(z) \neq 0, \sigma(w) \neq 0$ and $e(z)$ and $e(w)$ are linearly independent over $\mathbb{R}$.

(i) We define real numbers $c_{i, j, z, w}$ and $d_{i, j, z, w}$ so as to satisfy

$$
q_{i, j, z, w}=c_{i, j, z, w} e(z)+d_{i, j, z, w} e(w) .
$$

(ii) We define a (possibly empty) subspace of $M(n, m, \ell)$ by

$$
\begin{aligned}
S_{i, j, z, w} & =p_{i}^{-1}(z) \cap p_{j}^{-1}(w) \\
& =\left\{\left(a_{1}, \ldots, a_{n}\right) \in M(n, m, \ell) \mid a_{i}=z \text { and } a_{j}=w\right\} .
\end{aligned}
$$

If $S_{i, j, z, w}$ is not empty, we set $s_{i, j, z, w, v}=\left|q_{i, j, z, w}-v_{v}\right| / \ell$. Then it is clear that

$$
S_{i, j, z, w} \cong \prod_{\substack{1 \leq \nu \leq n \\ \nu \neq i, j}} P\left(m+1, s_{i, j, z, w, \nu}\right)
$$

Now we give our second result. See Remark 2.16 for the structure of $P(N, r)$ and Section 3.1 for a short review of Bott-Morse theory.

Theorem B Let $m, n \geq 2$ and assume that $\ell$ satisfies Assumption 2.10.

(i) We define a function $f: M(n, m, \ell) \rightarrow \mathbb{R}$ by

$$
f\left(a_{1}, \ldots, a_{n}\right)=\left|q\left(a_{1}, \ldots, a_{n}\right)\right|^{2}=\left|v_{1}+\ell \sum_{k=1}^{m} a_{1}^{k}\right|^{2} .
$$

Then $f$ is a Bott-Morse function.

(ii) The critical manifolds of $f$ are $f^{-1}(0)$ and the manifolds $R_{i, v}$ and $S_{i, j, z, w}$ in Definitions 2.12 and 2.13. We have

$$
f^{-1}(0) \cong(P(m+1,1 / \ell))^{n} .
$$


(iii) The index $\lambda$ of each critical manifold in (ii) is given as follows:

(a) We have $\lambda\left(f^{-1}(0)\right)=0$.

(b) We have

$$
\lambda\left(R_{i, v}\right)=\mu\left(v_{+}\right)-1,
$$

where $v_{+}=\left(v_{i} / \ell, z^{1}, \ldots, z^{m}\right) \in \mathbb{C}^{m+1}$.

(c) We have

$$
\lambda\left(S_{i, j, z, w}\right)= \begin{cases}\mu(z)+\mu(w)-2 & c_{i, j, z, w}>0 \text { and } d_{i, j, z, w}>0, \\ m-1+\mu(z)-\mu(w) & c_{i, j, z, w}>0 \text { and } d_{i, j, z, w}<0, \\ m-1-\mu(z)+\mu(w) & c_{i, j, z, w}<0 \text { and } d_{i, j, z, w}>0, \\ 2 m-\mu(z)-\mu(w) & c_{i, j, z, w}<0 \text { and } d_{i, j, z, w}<0 .\end{cases}
$$

Remark 2.14 We write (2-4) by

$$
0=q+c_{i, j, z, w}\left(-\frac{q-v_{i}}{\left|q-v_{i}\right|}\right)+d_{i, j, z, w}\left(-\frac{q-v_{j}}{\left|q-v_{j}\right|}\right),
$$

where $q=q_{i, j, z, w}$. Then $c_{i, j, z, w}>0$ if and only if $O$ and $v_{i}$ belong to the same half-plane determined by the line through $q$ and $v_{j}$. Hence the indices are summarized by Figure 2.

As an application of Theorem $\mathrm{B}$, we can compute $\chi(M(n, m, \ell))$. In particular, in Section 6 , we give a new proof of the formula of $\chi(P(2 m+1, r))$ :

Proposition 2.15 If $m$ is a positive integer and $r$ a positive real number such that $r / 2 \notin \mathbb{Z}$, then

$$
\chi(P(2 m+1, r))=2(-1)^{m+[r / 2]+1}\left(\begin{array}{c}
2 m-1 \\
m+[r / 2]
\end{array}\right) .
$$

Remark 2.16 It is well known that $P(N, r)$ has a singular point if $N-r$ is an odd integer and is a closed manifold of dimension $N-3$ otherwise. (See, for example, Hausmann [7] and Kapovich and Millson [10].) The groups $H_{*}(P(N, r) ; \mathbb{Z})$ are torsion free and the Poincaré polynomials are known. (See, for example, Farber and Schütz [6].) This formula (2-5) is given explicitly in Kamiyama [8, Theorem 3.4]. Note that if $r$ is not an odd integer, then

$$
\chi(P(2 m, r))=0
$$

because $P(2 m, r)$ is an odd-dimensional manifold. 

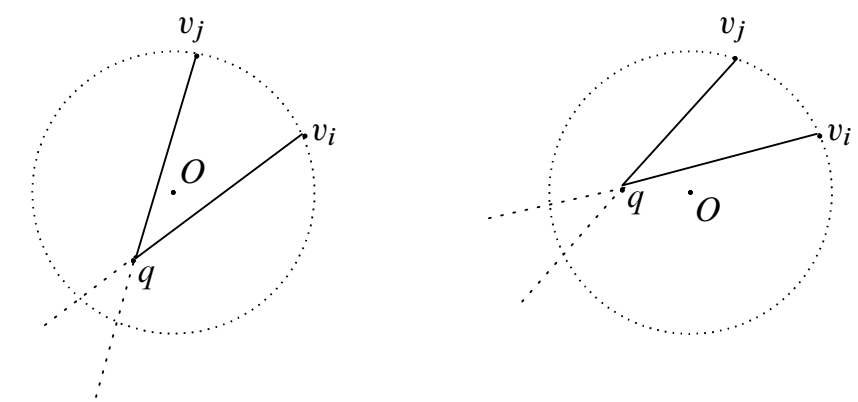

$$
\lambda=\mu(z)+\mu(w)-2
$$

$$
\lambda=m-1+\mu(z)-\mu(w)
$$
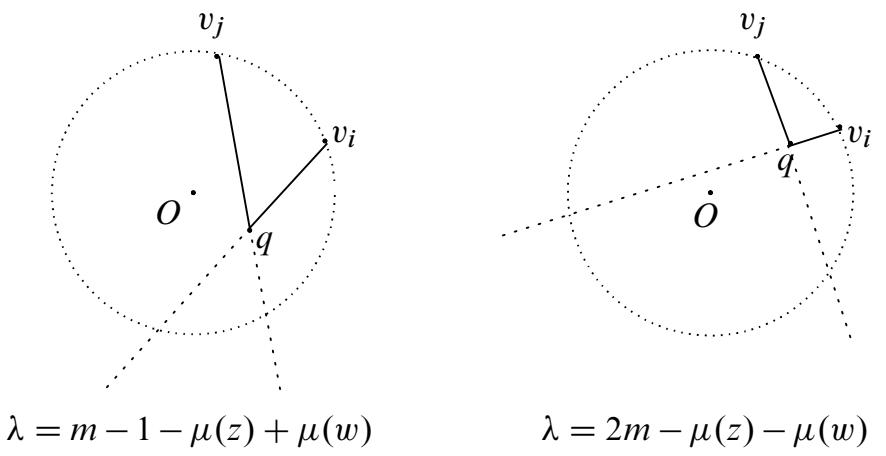

Figure 2: The indices $\lambda\left(S_{i, j, z, w}\right)$

For $n \geq 3$, we have the following:

\section{Theorem C Let $n \geq 3$.}

(i) If $m$ is odd and $\ell$ satisfies Assumption 2.10, then we have $\chi(M(n, m, \ell))=0$.

(ii) If $m$ is even, we have the following results.

(a) If $\ell$ satisfies the assumption of Theorem A(iv), namely, if $1 / m<\ell<\xi(n, m)$, then we have

$$
\chi(M(n, m, \ell))=(4-n) 2^{n-2} .
$$

(b) If $\ell>d_{n} / 2$, then

$$
\chi(M(n, m, \ell))=2^{n-2}\left((4-2 n) c_{m / 2}^{n}+n \sum_{\nu=1}^{m / 2}(-1)^{v}\left(\begin{array}{c}
m+1 \\
v
\end{array}\right) c_{\nu}^{n-1}\right)
$$

where we set $c_{v}=(-1)^{v-1}\left(\begin{array}{c}m-1 \\ v-1\end{array}\right)$. 
We give the asymptotic behavior of $\chi(M(n, m, \ell))$ in Proposition 5.2.

Remark 2.17 (i) In O'Hara [13], a Morse function on $M(n, 2, \ell)$ is constructed. In our paper [9], a slightly different Morse function on $M(n, 2, \ell)$ is constructed and it is shown that a similar function is a Bott-Morse function on the configuration space of a 2-leg machine in $\mathbb{R}^{d}$. The function $f$ in Theorem $\mathrm{B}$ is constructed by a similar idea to [9]. But since our linkage is a $m$-leg machine, the computations are much more difficult.

(ii) Theorem C(i) is clear when $n$ is odd because in this case, $M(n, m, \ell)$ is an odd-dimensional manifold.

Combining Theorem A(iv), Theorem C(i) and (ii)(a), and (2-5), we obtain a new proof of the following formula of $\chi\left(\mathcal{Z}_{P_{n}}\left(S^{m-2}\right)\right)$.

Corollary 2.18 We have

$$
\chi\left(\mathcal{Z}_{P_{n}}\left(S^{m-2}\right)\right)= \begin{cases}(4-n) 2^{n-2} & m \text { is even or } n=2, \\ 0 & n \geq 3 \text { and } m \text { is odd }\end{cases}
$$

Remark 2.19 The groups $H_{*}\left(\mathcal{Z}_{P_{n}}\left(S^{1}\right) ; \mathbb{Z}\right)$ are known by Buchstaber and Panov [4]. In [9, Theorem B], we gave a splitting of $\Sigma \mathcal{Z}_{P_{n}}\left(S^{m-2}\right)$. (See also Bahri, Bendersky, Cohen and Gitler [1].) From this, we have the following result: the groups $H_{*}\left(\mathcal{Z}_{P_{n}}\left(S^{m-2}\right) ; \mathbb{Z}\right)$ are torsion free such that the Poincaré polynomial is given as

$$
P_{t}\left(\mathcal{Z}_{P_{n}}\left(S^{m-2}\right)\right)=1+t^{n(m-2)+2}+\sum_{i=2}^{n-2}\left\{n\left(\begin{array}{c}
n-2 \\
i-1
\end{array}\right)-\left(\begin{array}{c}
n \\
i
\end{array}\right)\right\} t^{i(m-2)+1} .
$$

By Example 2.6(ii), $M(n, 2, \ell)$ is $S^{2}$ if and only if $n=2$ or 3 and $\ell<d_{n} / 2$. The following theorem asserts that a similar result holds for $m \geq 3$.

Theorem D Let $n \geq 2$ and assume $\ell$ satisfies Assumption 2.10. Then $M(n, m, \ell)$ is homeomorphic to a sphere if and only if $n=2$ or 3 and $1 / m<\ell<\xi(n, m)$.

\section{Preliminaries}

\subsection{A review of Bott-Morse theory}

We give a short review of Bott-Morse theory. For more details, see Bott [2] and McDuff and Salamon [12]. Let $M$ be a compact manifold. A smooth function $f: M \rightarrow \mathbb{R}$ 
is called a Bott-Morse function if its critical point set is a finite disjoint union of connected submanifolds called critical manifolds, and the Hessian of $f$ is fibrewise nondegenerate on the normal bundle of critical manifolds. An index $\lambda(C)$ of the critical manifold $C$ is the dimension of the negative eigenspace of the Hessian of $f$ on the normal bundle of $C$. A consequence of Bott-Morse theory is

$$
\sum_{C}(-1)^{\lambda(C)} \chi(C)=\chi(M)
$$

where the sum runs over all critical manifolds.

\subsection{Free robot arms}

Free robot arms are the most simple linkages. We recall some results from Walker [15] and Hausmann [7].

Theorem 3.1 [7] We fix $(m, \ell) \in \mathbb{N} \times \mathbb{R}_{>0}$. We define a map $\beta$ : $T^{m} \rightarrow \mathbb{C}$ by

$$
\beta(a)=\ell \sigma(a)=\ell \sum_{i=1}^{m} a^{i}
$$

and let

$$
\gamma=\left.\beta\right|_{V}: V=\beta^{-1}\left(\mathbb{R}_{>0}\right) \rightarrow \mathbb{R}
$$

be its restriction. Then the following results hold.

(i) The diagram

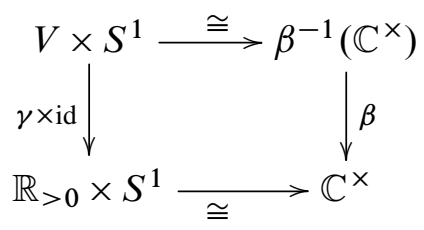

is commutative, where the horizontal arrows are given by the multiplication of complex numbers.

(ii) (a) $V$ is a codimension-1 submanifold of $T^{m}$ and the function $\gamma$ is a Morse function.

(b) The set of the critical points of $\gamma$ is $\{-1,1\}^{m} \cap V$.

(c) The index of the critical point $\varepsilon \in\{-1,1\}^{m} \cap V$ is $\mu(\varepsilon)-1$.

Proof This theorem is well known; it is a special case of [7, Theorems 3.1 and 3.2]. 
Since the map $\gamma$ does not have critical value greater than $\ell(m-2)$ except the maximum, we have the following.

Corollary 3.2 Over the annulus $A=\{z \in \mathbb{C}|\ell(m-2)<| z \mid \leq \ell m\}$, there exists a trivialization of the projection $\beta$

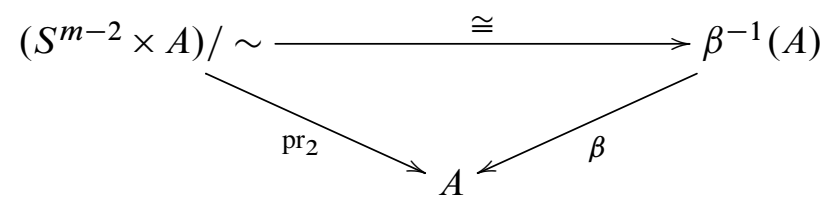

where $(x, z) \sim\left(x^{\prime}, z\right)$ if $|z|=\ell m$ and $\operatorname{pr}_{2}$ is the projection to the second factor.

Theorem 3.3 We fix $(m, \ell) \in \mathbb{N} \times \mathbb{R}_{>0}$ on the condition that $1 / \ell-m$ is not an even number. We define a function $g: T^{m} \rightarrow \mathbb{R}$ by

$$
g\left(z^{1}, \ldots, z^{m}\right)=\left|1+\ell \sum_{i=1}^{m} z^{i}\right|^{2} .
$$

Then the following results hold.

(i) The function $g$ is a Bott-Morse function.

(ii) The critical manifolds of $g$ are given as follows:

(a) $g^{-1}(0) \cong P(m+1,1 / \ell)$.

(b) $\{-1,1\}^{m}$.

(iii) The index of each critical manifold in (ii) is given as follows:

(a) $\lambda\left(g^{-1}(0)\right)=0$.

(b) For $\varepsilon \in\{-1,1\}^{m}$,

$$
\begin{array}{r}
\lambda(\varepsilon)=\mu\left(\varepsilon_{+}\right)-1 \\
\text { where } \varepsilon_{+}=\left(1 / \ell, \varepsilon^{1}, \ldots, \varepsilon^{m}\right) \in \mathbb{C}^{m+1} .
\end{array}
$$

Proof This theorem is well known, as [15, Proposition 3.3] and also obtained as a corollary of Theorem 3.1. Note that the condition that $1 / \ell-m$ is not an even number ensures that 0 is a regular value of the map $\left(z^{1}, \ldots, z^{m}\right) \mapsto 1+\ell \sum z^{i} \in \mathbb{C}$. 


\section{A Bott-Morse function on polygon spaces}

We consider certain functions on polygon spaces. First, we need the following:

Lemma 4.1 Let $m$ be a positive integer. For $1 \leq i \leq m$, let $\alpha_{i}$ and $\beta_{i}$ be elements of $\mathbb{R} \backslash\{0\}$. Consider the symmetric matrix defined by

$$
A=\operatorname{diag}\left(\alpha_{1}, \ldots, \alpha_{m}\right)+\left(\beta_{i} \beta_{j}\right) .
$$

We set $\mu=\sharp\left\{i \mid \alpha_{i}>0\right\}$ and

$$
D=1+\sum_{i=1}^{m} \frac{\beta_{i}^{2}}{\alpha_{i}}
$$

Then the signature of $A$ is

$$
\begin{cases}(\mu, m-\mu) & D>0 \\ (\mu, m-\mu-1) & D=0 \\ (\mu+1, m-\mu-1) & D<0 .\end{cases}
$$

Proof Without loss of generality, we assume $\alpha_{1}, \ldots, \alpha_{\mu}>0$ and $\alpha_{\mu+1}, \ldots, \alpha_{m}<0$. We set

$$
\phi_{i}=\sum_{j=1}^{i} \frac{\beta_{j}^{2}}{\alpha_{j}}, \quad \psi_{i}=\sum_{j=\mu+1}^{i} \frac{\beta_{j}^{2}}{\alpha_{j}} \quad \text { and } \quad \lambda=-\frac{1+\phi_{\mu}}{\psi_{m}} .
$$

Note that $\phi_{i}>0$ for $1 \leq i \leq \mu, \psi_{i}<0$ for $\mu+1 \leq i \leq m$ and $\lambda>0$. Let $U=\left(u_{1}, \ldots, u_{m}\right)$ be an $m \times m$ matrix whose $i^{\text {th }}$ column vector $u_{i}$ is given by

$$
u_{i}= \begin{cases}{ }^{\mathrm{t}}\left(\frac{\beta_{1}}{\alpha_{1}}, \ldots, \frac{\beta_{i}}{\alpha_{i}},-\frac{\phi_{i}}{\beta_{i+1}}, 0, \ldots, 0\right) & 1 \leq i \leq \mu-1, \\ { }^{\mathrm{t}}\left(\frac{\beta_{1}}{\alpha_{1}}, \ldots, \frac{\beta_{\mu}}{\alpha_{\mu}}, 0, \ldots, 0\right) & i=\mu, \\ { }^{(}\left(0, \ldots, 0, \frac{\beta_{\mu+1}}{\alpha_{\mu+1}}, \ldots, \frac{\beta_{i}}{\alpha_{i}},-\frac{\psi_{i}}{\beta_{i+1}}, 0, \ldots, 0\right) & \mu+1 \leq i \leq m-1, \\ { }^{(}\left(\frac{\beta_{1}}{\alpha_{1}}, \ldots, \frac{\beta_{\mu}}{\alpha_{\mu}}, \frac{\beta_{\mu+1} \lambda}{\alpha_{\mu+1}}, \ldots, \frac{\beta_{m} \lambda}{\alpha_{m}}\right) & i=m \text { (and } \mu<m) .\end{cases}
$$

It is easy to see that the matrix $U$ is invertible and a straightforward computation shows that ${ }^{\mathrm{t}} U A U=\operatorname{diag}\left(\gamma_{1}, \ldots, \gamma_{m}\right)$, where

$$
\gamma_{i}= \begin{cases}\phi_{i}+\left(\alpha_{i+1} / \beta_{i+1}^{2}\right) \phi_{i}^{2} & 1 \leq i \leq \mu-1, \\ \phi_{\mu}+\phi_{\mu}^{2} & i=\mu, \\ \psi_{i}+\left(\alpha_{i+1} / \beta_{i+1}^{2}\right) \psi_{i}^{2} & \mu+1 \leq i \leq m-1, \\ -\lambda\left(1+\phi_{\mu}+\psi_{m}\right) & i=m .\end{cases}
$$

Since $\gamma_{i}>0$ for $1 \leq i \leq \mu, \gamma_{i}<0$ for $\mu+1 \leq i \leq m-1, \gamma_{m}=-\lambda D$ and $\lambda>0$, we complete the proof. 
Theorem 4.2 We fix $(m, \ell, \zeta) \in \mathbb{N} \times \mathbb{R}_{>0} \times\left(S^{1} \backslash\{1\}\right)$ on the condition that

$$
\begin{gathered}
|(\zeta-1) / \ell| \\
1 / \ell-m, \\
|(\zeta-1) / \ell-m+2 k|-m \quad \text { for all } 0 \leq k \leq m
\end{gathered}
$$

are not even numbers. We set

$$
L=L(m, \ell, \zeta)=\left\{(z, w) \in T^{m} \times T^{m} \mid 1+\ell \sum_{i=1}^{m} z^{i}=\zeta+\ell \sum_{i=1}^{m} w^{i}\right\},
$$

where $z=\left(z^{1}, \ldots, z^{m}\right)$ and $w=\left(w^{1}, \ldots, w^{m}\right)$. We define a function $h: L \rightarrow \mathbb{R}$ by

$$
h(z, w)=\left|1+\ell \sum_{i=1}^{m} z^{i}\right|^{2}
$$

Then the following results hold.

(i) The space $L$ is a closed manifold of dimension $2 m-2$ and the function $h$ is a Bott-Morse function.

(ii) The critical manifolds of $h$ are given as follows:

(a) $h^{-1}(0) \cong P(m+1,1 / \ell)^{2}$.

(b) One of the following assertions holds:

(1) $z^{i}= \pm 1$ for all $1 \leq i \leq m$.

(2) $w^{i}= \pm \zeta$ for all $1 \leq i \leq m$.

In these cases, the critical manifold is of the form

$$
\left\{\left(z^{1}, \ldots, z^{m}\right)\right\} \times P(m+1, r) \text { or } P(m+1, r) \times\left\{\left(w^{1}, \ldots, w^{m}\right)\right\},
$$

where $r=|(\zeta-1) / \ell-m+2 k|$ for some integer $0 \leq k \leq m$.

(c) Both of the following assertions hold:

- $z^{i}= \pm z^{1}$ for all $1<i \leq m$.

- $w^{i}= \pm w^{1}$ for all $1<i \leq m$.

Note that, under the assumption of this theorem, each point of $L$ satisfies at most one of these three cases.

(iii) The index of each critical manifold in (ii) is given as follows:

(a) $\lambda\left(h^{-1}(0)\right)=0$.

(b) (1) $\lambda=\mu\left(z_{+}\right)-1$, where $z_{+}=\left(1 / \ell, z^{1}, \ldots, z^{m}\right) \in \mathbb{C}^{m+1}$.

(2) $\lambda=\mu\left(w_{+}\right)-1$, where $w_{+}=\left(\zeta / \ell, w^{1}, \ldots, w^{m}\right) \in \mathbb{C}^{m+1}$. 
(c) Let $(z, w)$ be a critical point. Define real numbers $c$ and $d$ so as to satisfy

$$
1+\ell \sum_{i=1}^{m} z^{i}=c e(z)+d e(w) .
$$

Then the index of the critical point is given as

$$
\lambda(z, w)= \begin{cases}\mu(z)+\mu(w)-2 & c>0 \text { and } d>0, \\ m-1+\mu(z)-\mu(w) & c>0 \text { and } d<0, \\ m-1-\mu(z)+\mu(w) & c<0 \text { and } d>0, \\ 2 m-\mu(z)+\mu(w) & c<0 \text { and } d<0 .\end{cases}
$$

Proof We construct an atlas of $L$. We set

$$
\alpha_{j}(z)=1+\ell \sum_{k \neq j} z^{k} \quad \text { and } \quad \beta_{j}(w)=\zeta+\ell \sum_{k \neq j} w^{k} .
$$

For $1 \leq j, k \leq m$, we set

$$
\begin{aligned}
& \mathcal{U}_{j, k}=\left\{(z, w) \in L \mid z^{j} \neq \pm w^{k}\right\}, \\
& \mathcal{U}_{j, k}^{+}=\left\{(z, w) \in L \mid \Im\left(\left(\alpha_{j}-\beta_{k}\right) \bar{z}^{j}\right)>0\right\}, \\
& \mathcal{U}_{j, k}^{-}=\left\{(z, w) \in L \mid \Im\left(\left(\alpha_{j}-\beta_{k}\right) \bar{z}^{j}\right)<0\right\} .
\end{aligned}
$$

We easily see that, under the condition (4-1),

$$
L=\bigcup_{j, k} \mathcal{U}_{j, k} \quad \text { and } \quad \mathcal{U}_{j, k}=\mathcal{U}_{j, k}^{+} \cup \mathcal{U}_{j, k}^{-},
$$

whence $\mathcal{U}_{j, k}^{ \pm}$is an atlas of $L$. For example, a local coordinate on $\mathcal{U}_{1,1}^{ \pm}$is given by $\left(\theta_{2}, \ldots, \theta_{m}, \tau_{2}, \ldots, \tau_{m}\right)$, where we set

$$
z^{j}=e^{\sqrt{-1} \theta_{j}} \quad \text { and } \quad w^{j}=e^{\sqrt{-1} \tau_{j}} .
$$

We define $q: L \rightarrow \mathbb{C}$ by

$$
q(z, w)=1+\ell \sum_{i=1}^{m} z^{i}=\zeta+\ell \sum_{i=1}^{m} w^{i} .
$$

On $\mathcal{U}_{1,1}^{ \pm}$, we differentiate the equations

$$
\left|q-\alpha_{1}\right|^{2}=\ell^{2} \text { and } \quad\left|q-\beta_{1}\right|^{2}=\ell^{2} .
$$

Then we have

$$
\left\langle q_{\theta_{j}}, z^{1}\right\rangle=\ell\left\langle\sqrt{-1} z^{j}, z^{1}\right\rangle \text { and }\left\langle q_{\theta_{j}}, w^{1}\right\rangle=0
$$


where $\langle\cdot, \cdot\rangle$ is the Euclidean inner product in $\mathbb{C}=\mathbb{R}^{2}$.

Now we prove that the manifolds given in (ii) exhaust all critical manifolds of $h$.

(1) One can see that the condition (4-2) ensures that $0 \in \mathbb{C}$ is a regular value of the map $q$. Then it is clear that $h^{-1}(0)=q^{-1}(0)$ is a critical manifold. This is the case of (ii)(a).

Let $(z, w)$ be a critical point such that $h(z, w) \neq 0$. By symmetry, we may assume that $(z, w) \in \mathcal{U}_{1,1}$.

(2) If $q_{\theta_{j}}=q_{\tau_{j}}=0$ for all $2 \leq j \leq m$, then the first equation of (4-5) and a similar equation for $q_{\tau_{j}}$ tell us that $z^{j}= \pm z^{1}$ and $w^{j}= \pm w^{1}$. This is the case of (ii)(c).

(3) Assume that $q_{\theta_{j}} \neq 0$ for some $j$. Since $(z, w)$ is a critical point, we have $q_{\theta_{j}} \perp q$. This and the second equation of (4-5) tells us that $q \| w^{1}$. It follows that $q_{\tau_{k}}=0$ hence $w^{k}= \pm w^{1}$ for all $k$. (Otherwise, we have $q \| z^{1}$ and contradicts the fact that $(z, w) \in \mathcal{U}_{1,1}$.) What is more, the fact that $q=\zeta+\ell \sum w^{k}$ implies that $w^{1}= \pm \zeta$. One can easily show that, under the condition (4-3), for a fixed $w_{0} \in\{-\zeta, \zeta\}^{m}$, the subspace $\left\{(z, w) \in L \mid w=w_{0}\right\}$ is a submanifold of codimension $m$. This is the second case of (ii)(b).

(4) The case that $q_{\tau_{j}} \neq 0$ for some $j$ can be treated in the same way as in 3 .

Next we prove (iii). Part (iii)(a) is clear and (iii)(b) is an immediate consequence of Theorem 3.3(iii)(b). We prove (iii)(c). Note that $(z, w) \in \mathcal{U}_{1,1}$. We define $\varepsilon, \delta \in$ $\{-1,1\}^{m}$ by

$$
z^{j}=\varepsilon^{j} e(z) \text { and } w^{j}=\delta^{j} e(w) .
$$

Note that $\mu(z)=\mu(\varepsilon)$ and $\mu(w)=\mu(\delta)$. We claim that

$$
\begin{aligned}
\left(\frac{h_{s t}}{2}\right)=-c\left(\operatorname{diag}\left(\ell \varepsilon^{2}, \ldots, \ell \varepsilon^{m}\right)\right. & \left.+\frac{1}{\ell \varepsilon^{1}}\left(\ell \varepsilon^{j} \ell \varepsilon^{k}\right)\right) \\
& \oplus-d\left(\operatorname{diag}\left(\ell \delta^{2}, \ldots, \ell \delta^{m}\right)+\frac{1}{\ell \delta^{1}}\left(\ell \delta^{j} \ell \delta^{k}\right)\right) .
\end{aligned}
$$

In order to prove this, note that

$$
h_{s t}=2\left(c\left\langle q_{s t}, e(z)\right\rangle+d\left\langle q_{s t}, e(w)\right\rangle\right) .
$$

We compute $\left\langle q_{s t}, e(z)\right\rangle$ for $s=t=\theta_{j}$. By differentiating the first equation of (4-4) twice, we have

$$
\left\langle q_{s t}-\alpha_{1 s t}, \ell z^{1}\right\rangle+\left\langle q_{s}-\alpha_{1 s}, q_{t}-\alpha_{1 t}\right\rangle=0 .
$$

Since $\alpha_{1 s}=\sqrt{-1} \ell z^{j}, \alpha_{1 s t}=-\ell z^{j}, q_{s}=0$ and $z^{j}=\varepsilon^{j} e(z)$, we have

$$
\ell \varepsilon^{1}\left\langle q_{s t}, e(z)\right\rangle=-\ell^{2}-\ell^{2} \varepsilon^{j} \varepsilon^{1} \text {. }
$$


By similar computations, we have (4-6). Combining Lemma 4.1 and the facts that $\ell \sum_{j=1}^{m} \varepsilon^{j}=|q-1|>0$ and $\ell \sum_{j=1}^{m} \delta^{j}=|q-\zeta|>0$,
we see that

$$
\begin{aligned}
& \operatorname{sgn}\left(-\left(\operatorname{diag}\left(\ell \varepsilon^{2}, \ldots, \ell \varepsilon^{m}\right)+\frac{1}{\ell \varepsilon^{1}}\left(\ell \varepsilon^{j} \ell \varepsilon^{k}\right)\right)\right)=(m-\mu(\varepsilon), \mu(\varepsilon)-1), \\
& \operatorname{sgn}\left(-\left(\operatorname{diag}\left(\ell \delta^{2}, \ldots, \ell \delta^{m}\right)+\frac{1}{\ell \delta^{1}}\left(\ell \delta^{j} \ell \delta^{k}\right)\right)\right)=(m-\mu(\delta), \mu(\delta)-1) .
\end{aligned}
$$

Thus we obtain (iii)(c).

Finally, (i) is a consequence of (ii) and (iii).

Remark 4.3 The space $L$ is homeomorphic to $P(2 m+1,|\zeta-1| / \ell)$, where we consider the segment through 1 and $\zeta$ as the fixed edge. In reference to this, a BottMorse function $\tilde{h}: L \rightarrow \mathbb{R}$ defined by

$$
\tilde{h}\left(z^{1}, \ldots, z^{2 m}\right)=\left|z^{1}+z^{2}\right|^{2}
$$

is studied in Klyachko [11]. The computations of the indices are easier for $\tilde{h}$, but we cannot use this for the study of $M(n, m, \ell)$.

\section{Proofs of main theorems}

Proof of Theorem A It is straightforward to show (i).

Recall that a mechanical linkage is described as the pullback of endpoints maps from free arms. (See [15; 9].) In our case of spidery linkages, we have the following description. We define a map $\pi_{i}: T^{m} \rightarrow \mathbb{C}$ for each $1 \leq i \leq n$ by

$$
\pi_{i}(a)=v_{i}+\ell \sum_{k=1}^{m} a^{k}
$$

and we set

$$
\begin{array}{ll}
D_{i}=\pi_{i}\left(T^{m}\right)=\left\{q \in \mathbb{C}|| q-v_{i} \mid \leq \ell m\right\} \subset \mathbb{C}, & D=\bigcap_{i=1}^{n} D_{i}, \\
E_{i}=D \cap \partial D_{i}, & A_{i}=\pi_{i}^{-1}(D) \subset T^{m} .
\end{array}
$$

Note that $D=q(M(n, m, \ell))$ and $D \neq \varnothing$ if and only if $\ell \geq 1 / m$. 
Then we clearly have a pullback diagram:

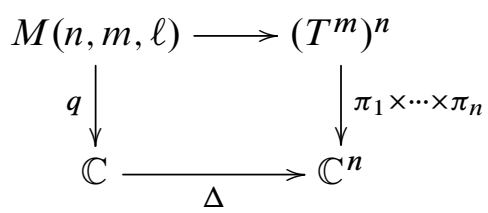

If $\ell$ is good, then it is easy to see that the maps $\pi_{1} \times \cdots \times \pi_{n}$ and $\Delta$ in the diagram are transversal, which implies (ii).

To see (iii), consider a similar pullback diagram:

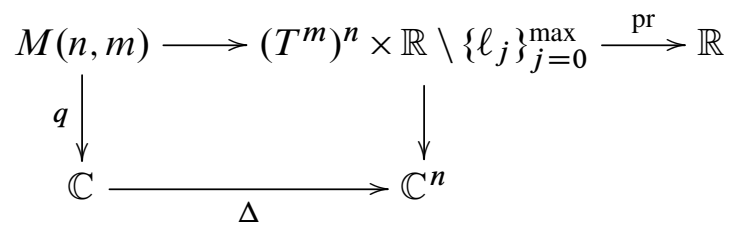

It is also straightforward to see the transversality of the pullback and that the horizontal composite $M(n, m) \rightarrow \mathbb{R}$ is a smooth proper function with no critical point.

With the aid of Corollary 3.2, the proof of (iv) is the same as that of [9, Theorem A]. We identify $D$ with the regular $n$-gon $P_{n}$ with edges $E_{1}, \ldots, E_{n}$. By Corollary 3.2, if $d\left(v_{i}, D\right)>\ell(m-2)$, then we have a trivialization of the projection $\pi_{i}$,

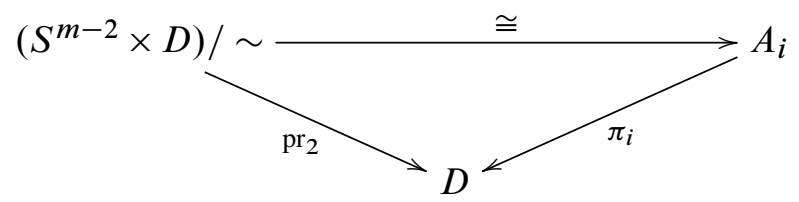

where $(x, q) \sim\left(x^{\prime}, q\right)$ if $q \in E_{i}$. Therefore,

$$
\begin{aligned}
M(n, m, \ell) & =A_{1} \times{ }_{D} A_{2} \times{ }_{D} \cdots \times{ }_{D} A_{n} \\
& \cong\left(S^{m-2}\right)^{n} \times D / \sim,
\end{aligned}
$$

where the equivalence relation $\sim$ is generated by the relation

$$
\left(x_{1}, \ldots, x_{n}, q\right) \sim\left(x_{1}, \ldots, *, \ldots, x_{n}, q\right) \quad \text { if } q \in E_{i},
$$

whence $M(n, m, \ell) \cong \mathcal{Z}_{P_{n}}\left(S^{m-2}\right)$.

Note that if $d\left(v_{i}, D\right)>0$, then

$$
d\left(v_{i}, D\right)= \begin{cases}2-\ell m, & n \text { is even } \\ 1+\cos (\pi / n)-\sqrt{m^{2} \ell^{2}-\sin ^{2}(\pi / n)}, & n \text { is odd }\end{cases}
$$


(The equal sign in the case when $i=1$ is attained by $z \in \mathbb{R} \subset \mathbb{C}$ such that $\left|z-v_{n / 2+1}\right|=$ $\ell m$ when $n$ is even and $\left|z-v_{(n+1) / 2}\right|=\left|z-v_{(n+3) / 2}\right|=\ell m$ when $n$ is odd.) Therefore, if $1 / m<\ell<\xi(n, m)$, then $d\left(v_{i}, D\right)>\ell(m-2)$ and we complete the proof of (iv).

Remark 5.1 In fact, in the diagram (5-2), the maps $\left(T^{m}\right)^{n} \times \mathbb{R} \rightarrow \mathbb{C}^{n}$ and $\Delta$ are transversal at a slightly wider class of $\ell$ and if $\ell \in L_{\mathrm{bad}}$, then $\ell$ is a critical value of the horizontal composite function.

Proof of Theorem B (ii) Recall that the function $f$ is defined by $f\left(a_{1}, \ldots, a_{n}\right)=$ $\left|q\left(a_{1}, \ldots, a_{n}\right)\right|^{2}$. In view of Theorem 3.1 and (5-1), we see $\left(a_{1}, \ldots, a_{n}\right) \in M(n, m, \ell)$ is a critical point of $q$ if and only if at least one arm is aligned. Therefore, by Assumption 2.10, $\left(a_{1}, \ldots, a_{n}\right) \in M(n, m, \ell)$ is a critical point of $f$ if and only if one of the following holds: $f\left(a_{1}, \ldots, a_{n}\right)=0$, one arm is aligned in the direction of some $v_{i}$ or two arms are aligned. Hence (ii) follows.

(iii)(a) By Assumption 2.10(i), $0 \in \mathbb{C}$ is a regular value of $q$, hence $f^{-1}(0)=q^{-1}(0)$ is a submanifold of codimension 2 whose index is 0 .

(c) For $1 \leq i<j \leq n$, we set

$$
\begin{aligned}
L_{i j} & =\left\{(z, w) \in T^{m} \times T^{m} \mid v_{i}+\ell \sigma(z)=v_{j}+\sigma(w)\right\} \\
M_{i j} & =\left\{\left(b_{1}, \ldots, \hat{b}_{i}, \ldots, \hat{b}_{j}, \ldots, b_{n}\right) \in\left(T^{m}\right)^{n-2} \mid v_{k}+\ell \sigma\left(b_{k}\right)=v_{l}+\ell \sigma\left(b_{l}\right)\right.
\end{aligned}
$$

for all $k, l\}$.

Then we have a pullback diagram

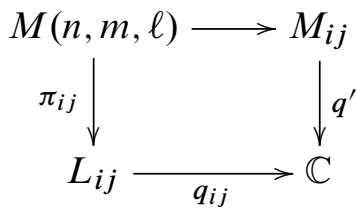

where the maps are the obvious ones. Note that $q=q_{i j} \circ \pi_{i j}$. Assume that two arms $a_{i}$ and $a_{j}$ of $\left(a_{1}, \ldots, a_{n}\right) \in M(n, m, \ell)$ are aligned. Since $\ell$ is good, $q_{i j}\left(a_{i}, a_{j}\right)$ is a regular value of $q^{\prime}$. Therefore, locally $q^{\prime}$ can be identified with a projection of the form $U \times V \rightarrow U$ for some neighborhood $U \subset \mathbb{C}$ of $q_{i j}\left(a_{i}, a_{j}\right)$ and $V \subset q^{-1}\left(q_{i j}\left(a_{i}, a_{j}\right)\right)$ of $\left(a_{1}, \ldots, \hat{a}_{i}, \ldots, \widehat{a_{j}}, \ldots, a_{n}\right)$ (or one may appeal to Ehresmann's fibration theorem to see that it is of the form $U \times S_{i, j, z, w} \rightarrow U$ ), whence so does $\pi_{i j}$ of the form $q_{i j}^{-1}(U) \times V \rightarrow q_{i j}^{-1}(U)$. Therefore the function $f$ can be identified locally with the composite

$$
q_{i j}^{-1}(U) \times V \longrightarrow q_{i j}^{-1}(U) \stackrel{q_{i j}}{\longrightarrow} \mathbb{C} \stackrel{|\cdot|^{2}}{\longrightarrow} \mathbb{R} .
$$


Clearly, $L_{i j}$ is diffeomorphic to the manifold $L$ in Theorem 4.2 and the function $\left|q_{i j}\right|^{2}$ is identified with $h$ in Theorem 4.2. Hence (c) follows from Theorem 4.2(c). One can show (b) similarly.

Part (i) is a consequence of (ii) and (iii).

Proof of Theorem C We use (2-5), (2-6), (3-1) and Theorem B. A proof of (2-5) is given in Section 6.

(i) Let $C$ be one of $f^{-1}(0), R_{i, v}$ or $S_{i, j, z, w}$. Since $n \geq 3, C$ has at least one $P(m+1, r)$ as a direct product factor. Since $m$ is odd, we have from (2-6) that $\chi(P(m+1, r))=0$. Hence $\chi(C)=0$. Using (3-1), we have $\chi(M(n, m, \ell))=0$.

(ii) Both (a) and (b) can be treated similarly. Since (a) is much easier, we consider (b).

We study the indices of critical manifolds in Theorem B when $\ell>d_{n} / 2$ and $m$ is even.

We set $c_{v}=(-1)^{v-1}\left(\begin{array}{c}m-1 \\ v-1\end{array}\right)$. Note that $c_{v}=(-1)^{m+1} c_{m+1-v}=-c_{m+1-v}$ since $m$ is even and, by (2-5), we have

$$
\chi(P(m+1, r))=-2 c_{m / 2+[r / 2]+1}=2 c_{m / 2-[r / 2]} .
$$

First, we have $f^{-1}(0) \cong(P(m+1,1 / \ell))^{n}$ with the index 0 . Since $0<1 / \ell<2$, the contribution of $f^{-1}(0)$ to the Euler characteristic is

$$
\chi(P(m+1,1 / \ell))^{n}=\left(2 c_{m / 2}\right)^{n}=2^{n} c_{m / 2}^{n} .
$$

Second, a short computation using Definition 2.12, $0<d_{n} / \ell<2$ and the triangle inequality for $q_{i, v}, v_{i}, v_{v}$ and $q_{i, v},-v_{i}, v_{v}$ tells us that

$$
0 \leq 2 \mu\left(v_{+}\right)-m-2<r_{i, v, v}<2 \mu\left(v_{+}\right)-m,
$$

whence $m / 2+\left[r_{i, v, v} / 2\right]+1=\mu\left(v_{+}\right)$for all $v \neq i$. Hence

$$
\chi\left(R_{i, v}\right)=\prod_{\nu \neq i} \chi\left(P\left(m+1, r_{i, v, v}\right)\right)=\left(-2 c_{\mu\left(v_{+}\right)}\right)^{n-1} .
$$

Note that $R_{i, v}=\varnothing$ if $\mu\left(v_{+}\right)=m+1$. By Theorem B, $\lambda\left(R_{i, v}\right)=\mu\left(v_{+}\right)-1$. Therefore the contribution of the $R_{i, v}$ is

$$
\begin{aligned}
n \sum_{\mu=m / 2+1}^{m}\left(\left(\begin{array}{c}
m \\
\mu-1
\end{array}\right)+\left(\begin{array}{c}
m \\
\mu
\end{array}\right)\right)(-1)^{\mu-1}\left(-2 c_{\mu}\right)^{n-1} \\
\quad=2^{n-1} n \sum_{\nu=1}^{m / 2}\left(\begin{array}{c}
m+1 \\
v
\end{array}\right)(-1)^{v} c_{v}^{n-1} .
\end{aligned}
$$

Third, the triangle inequality tells us that $S_{i, j, z, w} \neq \varnothing$ only when $\mu(z)=\mu(w)=: \mu$. Moreover, if $j \neq i+1(\bmod n)$, then $S_{i, j, z, w}=\varnothing$ if $\mu=m$. If $j=i+1(\bmod n)$ 
and $\mu=m$, then one of $S_{i, j, z, w}$ (whose $z$ and $w$ point "outside") is empty. Again by the triangle inequality, for all $v \neq i, j$, we have

$$
\begin{aligned}
& \begin{cases}2 \mu-m-2<s_{i, j, z, w, v}<2 \mu-m & \text { if }\left|q_{i, j, z, w}-v_{\nu}\right|<\left|q_{i, j, z, w}-v_{i}\right|, \\
2 \mu-m<s_{i, j, z, w, v}<2 \mu-m+2 & \text { if }\left|q_{i, j, z, w}-v_{\nu}\right|>\left|q_{i, j, z, w}-v_{i}\right|,\end{cases} \\
& m / 2+\left[s_{i, j, z, w, v} / 2\right]+1= \begin{cases}\mu & \text { if }\left|q_{i, j, z, w}-v_{\nu}\right|<\left|q_{i, j, z, w}-v_{i}\right|, \\
\mu+1 & \text { if }\left|q_{i, j, z, w}-v_{\nu}\right|>\left|q_{i, j, z, w}-v_{i}\right| .\end{cases}
\end{aligned}
$$

Hence

$$
\chi\left(S_{i, j, z, w}\right)=\prod_{\nu \neq i, j} \chi\left(P\left(m+1, s_{i, j, z, w, \nu}\right)\right)=(-2)^{n-2} c_{\mu}^{k} c_{\mu+1}^{n-2-k}
$$

for $k=j-i-1$ or $k=n-2-(j-i-1)$ and the index is given by $\lambda\left(S_{i, j, z, w}\right)=$ $\mu(z)+\mu(w)-2=2 \mu-2$ by Theorem B. The contribution of the $S_{i, j, z, w}$ is

$$
\begin{aligned}
& n \sum_{k=0}^{\lfloor(n-3) / 2\rfloor} \sum_{\mu=m / 2+1}^{m-1}\left(\begin{array}{l}
m \\
\mu
\end{array}\right)^{2}(-2)^{n-2}\left(c_{\mu}^{k} c_{\mu+1}^{n-2-k}+c_{\mu}^{n-2-k} c_{\mu+1}^{k}\right) \\
& +\rho(n) \sum_{\mu=m / 2+1}^{m-1}\left(\begin{array}{l}
m \\
\mu
\end{array}\right)^{2}(-2)^{n-2} c_{\mu}^{n / 2-1} c_{\mu+1}^{n / 2-1}+n(-2)^{n-2} c_{m}^{n-2} \\
& =2^{n-2} n\left(\sum_{\nu=2}^{m / 2}\left(\begin{array}{c}
m \\
v-1
\end{array}\right)^{2} \sum_{k=0}^{n-2} c_{v}^{k} c_{v-1}^{n-2-k}+c_{1}^{n-2}\right) \\
& =2^{n-2} n\left(\sum_{\nu=2}^{m / 2}\left(\begin{array}{c}
m \\
v-1
\end{array}\right)(-1)^{\nu-1}\left(c_{\nu}-c_{\nu-1}\right) \sum_{k=0}^{n-2} c_{\nu}^{k} c_{\nu-1}^{n-2-k}+c_{1}^{n-2}\right) \\
& =2^{n-2} n\left(\sum_{\nu=2}^{m / 2}\left(\begin{array}{c}
m \\
v-1
\end{array}\right)(-1)^{\nu-1}\left(c_{v}^{n-1}-c_{\nu-1}^{n-1}\right)+c_{1}^{n-2}\right) \\
& =2^{n-2} n \sum_{\nu=1}^{m / 2}\left(\begin{array}{c}
m \\
v-1
\end{array}\right)(-1)^{\nu-1}\left(c_{v}^{n-1}-c_{v-1}^{n-1}\right) \\
& =2^{n-2} n\left(\left(\begin{array}{c}
m \\
m / 2-1
\end{array}\right)(-1)^{m / 2-1} c_{m / 2}^{n-1}-\sum_{\nu=1}^{m / 2-1}\left(\begin{array}{c}
m+1 \\
v
\end{array}\right)(-1)^{v} c_{v}^{n-1}\right) \text {, }
\end{aligned}
$$

where $\rho(n)=n$ if $n$ is even and 0 if $n$ is odd. Combining (5-3), (5-4) and (5-5), using the equality

$$
\left(\begin{array}{c}
m+1 \\
m / 2
\end{array}\right)-\left(\begin{array}{c}
m \\
m / 2-1
\end{array}\right)=2\left(\begin{array}{c}
m-1 \\
m / 2-1
\end{array}\right)=(-1)^{m / 2-1} 2 c_{m / 2},
$$

we obtain the desired result. 
We study the asymptotic behavior of $\chi(M(n, m, \ell))$ of Theorem $\mathrm{C}(\mathrm{b})$.

Proposition 5.2 (i) For each fixed even $m$ and $\ell>d_{n} / 2$, we have

$$
\chi(M(n, m, \ell)) \sim(-1)^{((m n) / 2)+n+1} \frac{n(3 m+4)}{2 m+4}\left(\begin{array}{l}
m \\
\frac{m}{2}
\end{array}\right)^{n} \quad(n \rightarrow \infty) .
$$

(ii) For a fixed $n$, we have

$$
\begin{aligned}
&(-1)^{((m n) / 2)+n+1} \frac{n(3 m+4)}{2 m+4}\left(\begin{array}{c}
m \\
\frac{m}{2}
\end{array}\right)^{n} \\
& \sim(-1)^{((m n) / 2)+n+1} \frac{3 n}{2}\left(\frac{2^{m}}{\sqrt{(\pi m) / 2}}\right)^{n} \quad(m \rightarrow \infty) .
\end{aligned}
$$

Proof Recall that $c_{v}=(-1)^{v-1}\left(\begin{array}{c}m-1 \\ v-1\end{array}\right)$. Since

$$
(-1)^{v}\left(\begin{array}{c}
m+1 \\
v
\end{array}\right)=-(-1)^{v-1} \frac{m(m+1)}{v(m+1-v)}\left(\begin{array}{c}
m-1 \\
v-1
\end{array}\right)=-\frac{m(m+1)}{v(m+1-v)} c_{v}
$$

we see that

$$
\chi(M(n, m, \ell))=2^{n-1}\left(\left(2-\frac{(3 m+4) n}{m+2}\right) c_{m / 2}^{n}-\frac{m(m+1) n}{2} \sum_{\nu=1}^{m / 2-1} \frac{c_{v}^{n}}{v(m+1-v)}\right) .
$$

Clearly, the asymptotic behavior with respect to $n$ is determined by the first term. Using

$$
2 c_{m / 2}=(-1)^{(m / 2)-1} 2\left(\begin{array}{c}
m-1 \\
m / 2-1
\end{array}\right)=(-1)^{(m / 2)-1}\left(\begin{array}{c}
m \\
m / 2
\end{array}\right),
$$

(i) follows. Part (ii) follows from Stirling's formula.

Proof of Theorem D If $\ell$ satisfies $1 / m<\ell<\xi(n, m)$, then by Theorem A(iv), $M(n, m, \ell) \cong \mathcal{Z}_{P_{n}}\left(S^{m-2}\right)$. If $n=2$ or 3 , this is a sphere since, by definition, $\mathcal{Z}_{P_{2}}\left(S^{m-2}\right) \cong \Sigma S^{m-2} * S^{m-2}$ and $\mathcal{Z}_{P_{3}}\left(S^{m-2}\right)=S^{m-2} * S^{m-2} * S^{m-2}$.

Conversely, assume $M(n, m, \ell)$ is a sphere. We consider by case of the parity of $n$.

If $n$ is even, then $M(n, m, \ell)$ is an even sphere hence $\chi(M(n, m, \ell))=2$. It is easy to prove from Theorem B and Proposition 2.15 that $\chi(M(n, m, \ell))$ is divisible by $2^{n-2}$, whence $n=2$. Since $M(2, m, \ell) \cong P(2 m+1,2 / \ell)$, again by Proposition 2.15, we see that $\frac{1}{m}<\ell<\xi(n, m)$.

Next we consider the case of odd $n$. By Smith's theorem (see, for example, Bredon [3, page 129]), the fixed-point set of an involution acting on a sphere is a mod-2 
homology sphere. Hence, in order to complete the proof, it will suffice to construct an involution of $M(n, m, \ell)$ such that the fixed-point set is not a mod-2 homology sphere.

We define an involution of $M(n, m, \ell)$ by

$$
\sigma\left(a_{1}, \ldots, a_{n}\right)=\left(b_{1}, \ldots, b_{n}\right),
$$

where $b_{i}=\bar{a}_{n+2-i}$ for $1 \leq i \leq n$ with the convention $n+1=1$. If $\left(a_{1}, \ldots, a_{n}\right)$ is an element of the fixed point set, then we have $a_{1}^{k}= \pm 1(1 \leq k \leq m)$ and $a_{i}=\bar{a}_{n+2-i}$. Hence, similarly to $R_{1, \varepsilon}$ in Definition 2.12 , each connected component of the fixed point set is of the form

$$
F_{\varepsilon}(\ell):=\prod_{\nu=2}^{(n+1) / 2} P\left(m+1, r_{1, \varepsilon, \nu}\right)
$$

for some $\varepsilon \in\{-1,1\}^{m}$.

If $n \geq 5$, we take $\varepsilon$ to be $(-1,-1, \ldots,-1)$. By the triangle inequality, we see that $r_{1, \varepsilon, v}<m$ and by Assumption 2.10(ii), $m+1-r_{1, \varepsilon, v}$ is not an odd integer. Hence each factor $P\left(m+1, r_{1, \varepsilon, v}\right)$ is a closed orientable manifold of dimension $m-2$, which is $S^{0}$ in the case $m=2$. Therefore $F_{\varepsilon}(\ell)$ is a product of at least two such manifolds, hence is not a mod- 2 homology sphere.

If $\ell>\xi(n, m)$, then $d\left(v_{i}, D\right)<\ell(m-2)$ hence we see that $F_{\varepsilon}(\ell)$ is not empty for $\varepsilon=(1,-1, \ldots,-1),(-1,1,-1, \ldots,-1)$ and $(-1,-1, \ldots,-1)$. Therefore the fixed point set has at least three connected components, hence is not a mod-2 homology sphere.

\section{The case $n=2$}

We compute $\chi(P(2 m+1, r))$ using Theorem 4.2 and give a proof of Proposition 2.15. It is easy to see that when $N$ is even and $r$ is not an odd integer, then $P(N, r)$ is an odd-dimensional closed orientable manifold, hence $\chi(P(N, r))=0$ in this case and (2-6) holds. Clearly the formula holds when $N=3$. In order to prove by induction, we fix $m$ and assume that (2-5) holds for all odd $N \leq 2 m-1$.

We identify $P(2 m+1, r)$ with $L=L(m, 2 / r,-1)$ of Theorem 4.2. When $r$ is not an even integer, then it is straightforward to see that $\ell=2 / r$ and $\zeta=-1$ satisfy the assumption of Theorem 4.2.

Recall that, by comparing coefficients of

$$
(1-x)^{m-1}=(1-x)^{m}(1-x)^{-1} \text { and }(1-x)^{2 m-1}=(1-x)^{m}(1-x)^{m-1},
$$


we have identities

$$
\begin{aligned}
(-1)^{n}\left(\begin{array}{c}
m-1 \\
n
\end{array}\right) & =\sum_{\nu \leq n}(-1)^{v}\left(\begin{array}{c}
m \\
v
\end{array}\right)=-\sum_{v>n}(-1)^{v}\left(\begin{array}{c}
m \\
v
\end{array}\right), \\
\left(\begin{array}{c}
m-1 \\
n
\end{array}\right)^{2} & =-\sum_{\substack{\mu>n \\
v \leq n}}(-1)^{\mu+v}\left(\begin{array}{c}
m \\
\mu
\end{array}\right)\left(\begin{array}{c}
m \\
v
\end{array}\right), \\
(-1)^{n}\left(\begin{array}{c}
2 m-1 \\
n
\end{array}\right) & =\sum_{\mu+v \leq n}(-1)^{\mu+v}\left(\begin{array}{c}
m \\
\mu
\end{array}\right)\left(\begin{array}{c}
m \\
v
\end{array}\right)=-\sum_{\mu+v>n}(-1)^{\mu+v}\left(\begin{array}{c}
m \\
\mu
\end{array}\right)\left(\begin{array}{c}
m \\
v
\end{array}\right) .
\end{aligned}
$$

For an even integer $m$ and a subset $X \subset \mathbb{Z} \times \mathbb{Z}$, we set

$$
\Sigma_{X}=\sum_{(i, j) \in X}(-1)^{i+j}\left(\begin{array}{c}
m \\
i+m / 2
\end{array}\right)\left(\begin{array}{c}
m \\
j+m / 2
\end{array}\right)
$$

and define subsets of $\mathbb{Z} \times \mathbb{Z}$ by

$$
\begin{aligned}
S & =\{(i, j) \mid j<-r / 4<i\}, \\
T_{1} & =\{(i, j) \mid i-j>r / 2 \text { and } j<-r / 2\}, \\
T_{2} & =\{(i, j) \mid i-j>r / 2 \text { and }-r / 2<j<-r / 4\}, \\
T_{3} & =\{(i, j) \mid i-j>r / 2 \text { and }-r / 4<j\}, \\
U & =\{(i, j) \mid i+j>r / 2 \text { and }-r / 2<i-j<r / 2\} .
\end{aligned}
$$

In our situation, the critical manifolds and indices of Theorem 4.2 are given as follows:

$$
\begin{aligned}
& h^{-1}(0) \cong P(m+1,1 / \ell)^{2}=P(m+1, r / 2)^{2}, \\
& \chi(P(m+1, r / 2))= \begin{cases}2(-1)^{m / 2+[r / 4]+1}\left(\begin{array}{c}
m-1 \\
m / 2+[r / 4]
\end{array}\right) & m \text { is even, } \\
0 & m \text { is odd } .\end{cases}
\end{aligned}
$$

Note that

$$
\begin{aligned}
\left(\begin{array}{c}
m-1 \\
m / 2+[r / 4]
\end{array}\right)^{2}=\left(\begin{array}{c}
m-1 \\
m / 2-[r / 4]-1
\end{array}\right)^{2} & =-\sum_{\substack{\mu>m / 2-[r / 4]-1 \\
v \leq m / 2-[r / 4]-1}}(-1)^{\mu+v}\left(\begin{array}{l}
m \\
\mu
\end{array}\right)\left(\begin{array}{c}
m \\
v
\end{array}\right) \\
& =-\sum_{\substack{\mu-m / 2>-r / 4 \\
v-m / 2<-r / 4}}(-1)^{\mu+v}\left(\begin{array}{c}
m \\
\mu
\end{array}\right)\left(\begin{array}{c}
m \\
v
\end{array}\right)=-\Sigma_{S} .
\end{aligned}
$$

Hence, the contribution of $h^{-1}(0)$ is

$$
\begin{cases}-4 \Sigma_{S} & m \text { is even } \\ 0 & m \text { is odd. }\end{cases}
$$


(b) Critical manifolds of Theorem 4.2(ii)(b)(1) are indexed by elements of $\{-1,1\}^{m}$ and are of the form $P(m+1, s)$ for some positive real number $s$.

If $m$ is odd, then $\chi(P(m+1, s))=0$.

Consider the case when $m$ is even. For $z \in\{-1,1\}^{m}$, we set $v=\sharp\left\{j \mid z^{j}=1\right\}$. Then the length $s$ is given by $s=|2 v-m+r|$. Since

$$
[s / 2]= \begin{cases}-v+m / 2-[r / 2]-1 & \text { if } v-m / 2<-r / 2, \\ v-m / 2+[r / 2] & \text { if } v-m / 2>-r / 2,\end{cases}
$$

we have

$$
\chi(P(m+1, s))= \begin{cases}2(-1)^{m-v-[r / 2]}\left(\begin{array}{c}
m-1 \\
m-v-[r / 2]-1
\end{array}\right) & \text { if } v-m / 2<-r / 2, \\
2(-1)^{v+[r / 2]+1}\left(\begin{array}{c}
m-1 \\
v+[r / 2]
\end{array}\right) & \text { if } v-m / 2>-r / 2 .\end{cases}
$$

Since $q / \ell=2 v-m+r / 2$, we have

$$
\mu\left(z_{+}\right)= \begin{cases}m-v & \text { if } v-m / 2<-r / 4 \\ v+1 & \text { if } v-m / 2>-r / 4\end{cases}
$$

therefore

$$
(-1)^{\mu-1} \chi(P(m+1, s))= \begin{cases}2(-1)^{[r / 2]+1}\left(\begin{array}{c}
m-1 \\
v+[r / 2]
\end{array}\right) & \text { if } v-m / 2<-r / 2, \\
2(-1)^{[r / 2]}\left(\begin{array}{c}
m-1 \\
v+[r / 2]
\end{array}\right) & \text { if }-r / 2<v-m / 2<-r / 4, \\
2(-1)^{[r / 2]+1}\left(\begin{array}{c}
m-1 \\
v+[r / 2]
\end{array}\right) & \text { if }-r / 4<v-m / 2 .\end{cases}
$$

Since

$$
\left(\begin{array}{c}
m-1 \\
v+[r / 2]
\end{array}\right)=(-1)^{v+[r / 2]+1} \sum_{\mu>v+[r / 2]}(-1)^{\mu}\left(\begin{array}{l}
m \\
\mu
\end{array}\right)=(-1)^{[r / 2]+1} \sum_{\mu>v+r / 2}(-1)^{\mu+v}\left(\begin{array}{l}
m \\
\mu
\end{array}\right)
$$

the contribution of critical manifolds of Theorem 4.2(ii)(b)(1) is

$$
\begin{aligned}
& 2\left(\sum_{\nu-m / 2<-r / 2}-\sum_{-r / 2<v-m / 2<-r / 4}+\sum_{-r / 4<v-m / 2}\right)(-1)^{[r / 2]+1}\left(\begin{array}{c}
m \\
v
\end{array}\right)\left(\begin{array}{c}
m-1 \\
v+[r / 2]
\end{array}\right) \\
& \quad=2\left(\sum_{\nu-m / 2<-r / 2}-\sum_{-r / 2<v-m / 2<-r / 4}+\sum_{-r / 4<v-m / 2}\right) \sum_{\mu>v+r / 2}(-1)^{\mu+v}\left(\begin{array}{l}
m \\
\mu
\end{array}\right)\left(\begin{array}{l}
m \\
v
\end{array}\right) \\
& =2\left(\Sigma_{T_{1}}-\Sigma_{T_{2}}+\Sigma_{T_{3}}\right) .
\end{aligned}
$$

By symmetry, the contribution of critical manifolds of Theorem 4.2(ii)(b)(2) is the same as that of (b)(i). Hence the contribution of (b) is

$$
\begin{cases}4\left(\Sigma_{T_{1}}-\Sigma_{T_{2}}+\Sigma_{T_{3}}\right) & m \text { is even, } \\ 0 & m \text { is odd. }\end{cases}
$$


(c) Let $(z, w)$ be critical points of Theorem 4.2(ii)(c) and we set $\mu(z)=\mu, \mu(w)=v$. Since there is a triangle of edge lengths $(4 \mu-2 m) / r,(4 v-2 m) / r$ and $2,(\mu, v)$ must belong to the set

$$
\begin{aligned}
U^{\prime} & =\{(\mu, v) \in \mathbb{N} \times \mathbb{N} \mid m / 2<\mu, v \leq m \text { and }|\mu-v|<r / 2<\mu+v-m\} \\
& =\{(\mu, v) \in \mathbb{N} \times \mathbb{N} \mid \mu, v \leq m,-r / 2<\mu-v<r / 2 \text { and } m+r / 2<\mu+v\} .
\end{aligned}
$$

Hence, the contribution is

$$
\begin{cases}2 \Sigma_{U} & m \text { is even, } \\
2 \sum_{(\mu, v) \in U^{\prime}}(-1)^{\mu+v}\left(\begin{array}{c}
m \\
\mu
\end{array}\right)\left(\begin{array}{l}
m \\
v
\end{array}\right) & m \text { is odd. }\end{cases}
$$

If $m$ is odd, via the symmetry $(-1)^{i}\left(\begin{array}{c}m \\ i\end{array}\right)=-(-1)^{m-i}\left(\begin{array}{c}m \\ m-i\end{array}\right)$, one easily sees that the sum of (6-1), (6-2) and (6-3) is

$$
\begin{aligned}
2 \sum_{(\mu, v) \in U^{\prime}}(-1)^{\mu+v}\left(\begin{array}{c}
m \\
\mu
\end{array}\right)\left(\begin{array}{c}
m \\
v
\end{array}\right) & =2 \sum_{\mu+v>m+[r / 2]}(-1)^{\mu+v}\left(\begin{array}{c}
m \\
\mu
\end{array}\right)\left(\begin{array}{c}
m \\
v
\end{array}\right) \\
& =2(-1)^{m+[r / 2]+1}\left(\begin{array}{c}
2 m-1 \\
m+[r / 2]
\end{array}\right)
\end{aligned}
$$

as desired.

Consider the case when $m$ is even. The sum of (6-1), (6-2) and (6-3) is

$$
-4 \Sigma_{S}+4\left(\Sigma_{T_{1}}-\Sigma_{T_{2}}+\Sigma_{T_{3}}\right)+2 \Sigma_{U}
$$

Note: $(-1)^{i+j}\left(\begin{array}{c}m \\ i+m / 2\end{array}\right)\left(\begin{array}{c}m \\ j+m / 2\end{array}\right)$ is invariant under transformations $(i, j) \rightarrow( \pm i, \pm j)$ and $(i, j) \rightarrow(j, i)$, hence so is $\Sigma_{X}$.

Decompose the sets $S, T_{1}$ and $T_{3}$ into the following sets:

$$
\begin{aligned}
S & =S_{1} \cup S_{2} \cup S_{3} \\
S_{1} & =S \cap T_{1} \\
S_{2} & =\{(i, j) \mid i+j>-r / 2 \text { and }-r / 2<j<-r / 4\} \\
S_{3} & =\{(i, j) \in S \mid i+j<-r / 2 \text { and } i-j<r / 2\} \\
& =\{(i, j)|-r / 4<i<-| j+r / 2 \mid\} \\
T_{1}^{2} & =T_{1} \backslash S_{1}=\{(i, j) \mid i-j>r / 2 \text { and } i<-r / 4\} \\
T_{3} & =T_{3}^{1} \cup T_{3}^{2}
\end{aligned}
$$




$$
\begin{aligned}
T_{3}^{1} & =\left\{(i, j) \in T_{3} \mid i+j<r / 2\right\} \\
& =\{(i, j)|-r / 4<j<-| i-r / 2 \mid\} \\
T_{3}^{2} & =\left\{(i, j) \in T_{3} \mid i+j>r / 2\right\} \\
& =\{(i, j) \mid i+j>r / 2, i-j>r / 2 \text { and }-r / 4<j\}
\end{aligned}
$$

(see Figure 3).

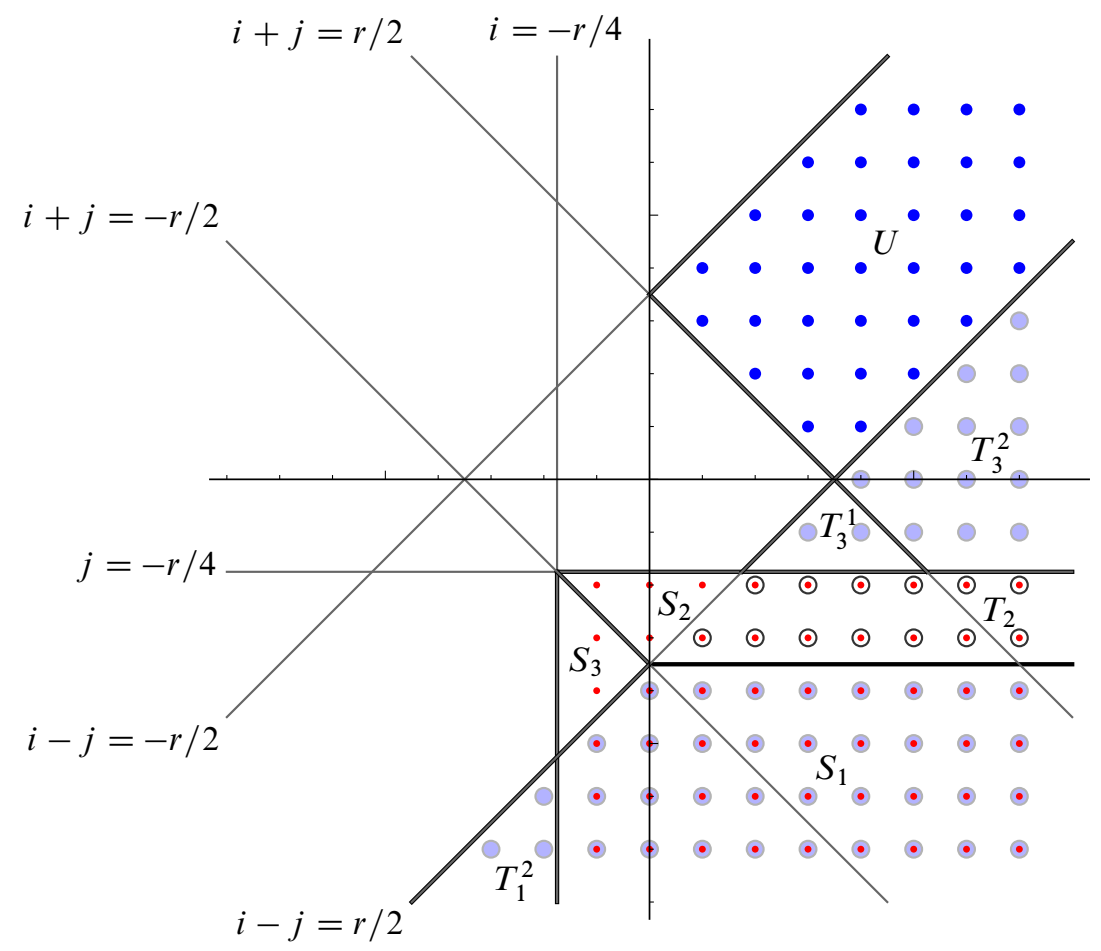

Figure 3: $m=14$ and $r=7$

Let

$$
\begin{aligned}
\bar{T}_{2} & =\left\{(i, j) \mid(-i, j) \in T_{2}\right\} \\
& =\{(i, j) \mid i+j<-r / 2 \text { and }-r / 2<j<-r / 4\} ;
\end{aligned}
$$

then we have

$$
S_{2} \cup \bar{T}_{2}=\{(i, j) \mid-r / 2<j<-r / 4\}, \quad S_{2} \cap \bar{T}_{2}=\varnothing,
$$

hence

$$
\Sigma_{S_{2}}+\Sigma_{T_{2}}=\Sigma_{S_{2}}+\Sigma_{\bar{T}_{2}}=\Sigma_{-r / 2<j<-r / 4}=0 .
$$


Note that $(i, j) \in S_{3}$ if and only if $(-j, i) \in T_{3}^{1}$, hence

$$
\Sigma_{S_{3}}=\Sigma_{T_{3}^{1}}
$$

Let

$$
\bar{T}_{1}^{2}=\left\{(i, j) \mid(j,-i) \in T_{1}^{2}\right\}
$$

then

$$
\Sigma_{T_{1}^{2}}=\Sigma_{\bar{T}_{1}^{2}}
$$

and we see that $\bar{T}_{1}^{2},{ }^{t} \bar{T}_{1}^{2}, T_{3}^{2},{ }^{t} T_{3}^{2}$ and $U$ are pairwise disjoint and

$$
\bar{T}_{1}^{2} \cup{ }^{t} \bar{T}_{1}^{2} \cup T_{3}^{2} \cup{ }^{t} T_{3}^{2} \cup U=\{(i, j) \mid i+j>[r / 2]\}
$$

whence

$$
\begin{aligned}
-4 \Sigma_{S}+4\left(\Sigma_{T_{1}}-\right. & \left.\Sigma_{T_{2}}+\Sigma_{T_{3}}\right)+2 \Sigma_{U} \\
= & 4\left(-\Sigma_{S_{1}}-\Sigma_{S_{2}}-\Sigma_{S_{3}}+\Sigma_{S_{1}}+\Sigma_{T_{1}^{2}}-\Sigma_{T_{2}}+\Sigma_{T_{3}^{1}}+\Sigma_{T_{3}^{2}}\right)+2 \Sigma_{U} \\
= & 4\left(\Sigma_{\bar{T}_{1}^{2}}+\Sigma_{T_{3}^{2}}\right)+2 \Sigma_{U} \\
= & 2\left(\Sigma_{\bar{T}_{1}^{2}}+\Sigma_{t} \bar{T}_{1}^{2}+\Sigma_{T_{3}^{2}}+\Sigma_{t} T_{3}^{2}+\Sigma_{U}\right) \\
= & 2 \Sigma_{i+j>[r / 2]} \\
= & 2 \sum_{i+j>[r / 2]}(-1)^{i+j}\left(\begin{array}{c}
m \\
i+m / 2
\end{array}\right)\left(\begin{array}{c}
m \\
j+m / 2
\end{array}\right) \\
= & 2 \sum_{\mu+v>m+[r / 2]}(-1)^{\mu+v}\left(\begin{array}{c}
m \\
\mu
\end{array}\right)\left(\begin{array}{c}
m \\
v
\end{array}\right) \\
= & 2(-1)^{m+[r / 2]+1}\left(\begin{array}{c}
2 m-1 \\
m+[r / 2]
\end{array}\right)
\end{aligned}
$$

as desired.

Remark 6.1 A certain variation of the function $h$ would give a simple proof of this formula, which will be treated elsewhere.

\section{References}

[1] A Bahri, M Bendersky, F R Cohen, S Gitler, The polyhedral product functor: A method of decomposition for moment-angle complexes, arrangements and related spaces, Adv. Math. 225 (2010) 1634-1668 MR2673742

[2] R Bott, Nondegenerate critical manifolds, Ann. of Math. 60 (1954) 248-261 MR0064399 
[3] G E Bredon, Introduction to compact transformation groups, Pure and Applied Math. 46, Academic Press, New York (1972) MR0413144

[4] V M Buchstaber, T E Panov, Torus actions and their applications in topology and combinatorics, Univ. Lecture Series 24, Amer. Math. Soc. (2002) MR1897064

[5] M W Davis, T Januszkiewicz, Convex polytopes, Coxeter orbifolds and torus actions, Duke Math. J. 62 (1991) 417-451 MR1104531

[6] M Farber, D Schütz, Homology of planar polygon spaces, Geom. Dedicata 125 (2007) 75-92 MR2322541

[7] J-C Hausmann, Sur la topologie des bras articulés, from: "Algebraic topology Poznań 1989”, (S Jackowski, B Oliver, K Pawałowski, editors), Lecture Notes in Math. 1474, Springer (1991) 146-159 MR1133898

[8] Y Kamiyama, Topology of equilateral polygon linkages, Topology Appl. 68 (1996) 13-31 MR1370925

[9] Y Kamiyama, S Tsukuda, The configuration space of the $n$-arms machine in the Euclidean space, Topology Appl. 154 (2007) 1447-1464 MR2310477

[10] M Kapovich, J Millson, On the moduli space of polygons in the Euclidean plane, J. Differential Geom. 42 (1995) 430-464 MR1366551

[11] A A Klyachko, Spatial polygons and stable configurations of points in the projective line, from: "Algebraic geometry and its applications", (A Tikhomirov, A Tyurin, editors), Aspects Math. E25, Vieweg, Braunschweig (1994) 67-84 MR1282021

[12] D McDuff, D Salamon, Introduction to symplectic topology, 2nd edition, Oxford Math. Monographs, Oxford Univ. Press (1998) MR1698616

[13] J O'Hara, The configuration space of planar spidery linkages, Topology Appl. 154 (2007) 502-526 MR2278700

[14] N Shvalb, M Shoham, D Blanc, The configuration space of arachnoid mechanisms, Forum Math. 17 (2005) 1033-1042 MR2195717

[15] K Walker, Configuration spaces of linkages, Bachelor's thesis, Princeton University (1985)

Department of Mathematics, University of the Ryukyus

Nishihara-Cho, Okinawa 903-0213, Japan

kamiyama@sci.u-ryukyu.ac.jp, tsukuda@math.u-ryukyu.ac.jp

Received: 24 January 2014 Revised: 8 June 2014 